\title{
Pitfalls in the Detection of Insulinomas With Glucagon-Like Peptide-1 Receptor Imaging
}

\author{
Kwadwo Antwi, MD, * Matthias Hepprich, MD,† Natasha A. Müller,ł Jean Claude Reubi, MD, $\S$ \\ Melpomeni Fani, PhD, * Christof Rottenburger, MD, *// Guillaume Nicolas, MD, *// Felix Kaul, MD, *// \\ Emanuel R. Christ, MD, PhD, $\dagger / /$ and Damian Wild, MD, PhD*//
}

\begin{abstract}
Purpose: Physiological pancreaticoduodenal uptake of radiolabeled exendin-4 in Brunner glands of the proximal duodenum is the most common pitfall for false interpretation of glucagon-like peptide-1 receptor (GLP-1R) imaging. The aim of this study was to analyze the pancreaticoduodenal uptake in GLP-1R PET/CT and SPECT/CT images and to identify additional potential reading pitfalls in patients with suspected insulinoma.

Methods: A post hoc analysis of a prospective study, including 52 consecutive patients, was performed. All patients underwent $1{ }^{68} \mathrm{Ga}$-exendin-4 $\mathrm{PET} / \mathrm{CT}$ and $2{ }^{111}$ In-exendin-4 SPECT/CT scans (4 and 72 hours postinjection) in a randomized crossover order. Three board-certified nuclear medicine physicians read all scans independently. They were unaware of other results. Reference standard was surgery with histopathological confirmation of an insulinoma/nesidioblastosis and normalization of blood glucose levels after surgery.
\end{abstract}

Results: There were no false-positive readings. However, there were a number of false-negative PET/CT and SPECT/CT readings, respectively: (1) due to false interpretation of uptake in the pancreaticoduodenal region (falsely interpreted as physiological uptake in Brunner glands instead of an insulinoma in $0.6 \%$ vs $9.0 \%$ ), (2) due to ectopic insulinoma ( $0 \%$ vs $2.6 \%$ ), (3) due to small insulinoma (1.9\% vs 5.1\%), (4) due to insulinoma overlap with kidneys $(1.9 \%$ vs $4.5 \%)$, and (5) due to nesidioblastosis ( $0.6 \%$ and $1.9 \%)$. Pitfalls were identified in all GLP-1R PET/CT and SPECT/CT scans.

Conclusions: Peripancreatic uptake, small size of an insulinoma, insulinoma overlap with kidneys, and presence of nesidioblastosis are potential pitfalls in GLP-1R imaging, which can lead to false reading results.

Key Words: ${ }^{68} \mathrm{Ga}$-exendin-4, ${ }^{111} \mathrm{In}$-exendin-4, Brunner glands, GLP-1R imaging, insulinoma, PET/CT, SPECT/CT

(Clin Nucl Med 2020;45: e386-e392)

Received for publication February 27, 2020; revision accepted April 19, 2020.

From the *Clinic of Radiology and Nuclear Medicine and $†$ Division of Endocrinology, Diabetology and Metabolism, University Hospital Basel; $\$$ Faculty of Clinical Medicine, University of Basel, Basel; §Division of Cell Biology and Experimental Cancer Research, Institute of Pathology, University of Bern, Bern; and $\|$ Center for Neuroendocrine and Endocrine Tumors, University Hospital Basel, Basel, Switzerland.

Conflicts of interest and sources of funding: The study was supported by the Swiss National Science Foundation (grant 320030-152938) and the Desirée and Niels Yde's Foundation (grant 389-12), which had no role in study design, data collection, analysis, interpretation, or writing of the report. No conflict of interest for all authors.

The study was approved by the regional scientific ethics committee, and all procedures performed in studies involving human participants were in accordance with the ethical standards of the regional scientific ethics committee and with the 1964 Helsinki Declaration and its later amendments or comparable ethical standards.

Informed consent was obtained from all individual participants included in the study.

Correspondence to: Damian Wild, MD, PhD, Clinic of Radiology and Nuclear Medicine, University Hospital Basel, Petersgraben 4, 4053 Basel, Switzerland. E-mail: damian.wild@usb.ch.

Copyright $(\mathrm{C} 2020$ Wolters Kluwer Health, Inc. All rights reserved.

ISSN: 0363-9762/20/4509-e386

DOI: 10.1097/RLU.0000000000003124

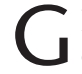
lucagon-like peptide-1 receptor (GLP-1R) imaging with PET or SPECT has become an essential diagnostic tool in the detection of benign insulinomas in patients with endogenous hyperinsulinemic hypoglycemia $(\mathrm{EHH}) .{ }^{1,2}$ Other indications include (i) disease assessment in malignant insulinoma, ${ }^{3,4}$ (ii) detection of clinically relevant lesions in patients with multiple endocrine neoplasia type $1(\mathrm{MEN}-1)$ and $\mathrm{EHH},{ }^{5}$ and (iii) detection of nesidioblastosis in adults. 1,6

In the first-ever prospectively executed GLP-1R study, ${ }^{111} \mathrm{In}$ DTPA-exendin-4 SPECT/CT performed significantly better than conventional imaging, that is, CT and/or MRI. ${ }^{1}$ Despite the high sensitivity of GLP-1R SPECT/CT, some erroneous readings results were encountered, partially misguiding the ensuing clinical procedures. For example, in 1 patient, ${ }^{111}$ In-DTPA-exendin-4 SPECT/ CT revealed a focal uptake in the pancreatic head adjacent to the duodenum, as well as in the mesentery of the small bowel both suggestive of an insulinoma. ${ }^{7}$ Because previously performed arterial calcium stimulation with venous sampling (ASVS) likewise displayed a pathological gradient in the area of the superior mesenteric artery, the patient subsequently received a duodenopancreatectomy. However, no insulinoma was visualized in the pancreatic head but in the mesentery of the small bowel consistent with the second ectopic focal ${ }^{111} \mathrm{In}$ DTPA-exendin-4 uptake. Immunohistochemistry of the resected duodenum showed positive GLP-1R staining of the Brunner glands (personal communication). This is in conjunction with previously published data that Brunner glands can overexpress the GLP-1R. This might be the most reasonable explanation for the falsepositive pancreaticoduodenal finding in this patient.

In view of the described pitfall, as well as the possible resulting clinical consequences, the aims of this study were to perform a post hoc analysis of our prospective acquired data to analyze pancreaticoduodenal uptake in ${ }^{111}$ In-DOTA-exendin-4 SPECT/CT and ${ }^{68}$ Ga-DOTA-exendin-4 PET/CT images and to identify additional pitfalls potentially leading to erroneous reading results.

\section{MATERIALS AND METHODS}

\section{Study Design and Patients}

A post hoc analysis of a prospective study, including 52 consecutive patients, was performed in order to identify and quantify pitfalls. Patients were included if they had biochemically proven EHH with neuroglycopenic symptoms and were excluded if the following conditions were present: (i) evidence of a malignant insulinoma on conventional imaging, (ii) pregnancy or breastfeeding in women, and (iii) renal insufficiency (serum creatinine $>140 \mu \mathrm{mol} / \mathrm{L}$ ).

The regional scientific ethics committee approved the study, and all patients provided written consent in accordance with provisions of the Declaration of Helsinki.

\section{Procedures}

All patients underwent $1 \mathrm{PET} / \mathrm{CT}$ with 43 to $106 \mathrm{MBq}{ }^{68} \mathrm{Ga}-$ DOTA-exendin-4 and 2 SPECT/CT scans with 52 to $111 \mathrm{MBq}$ 


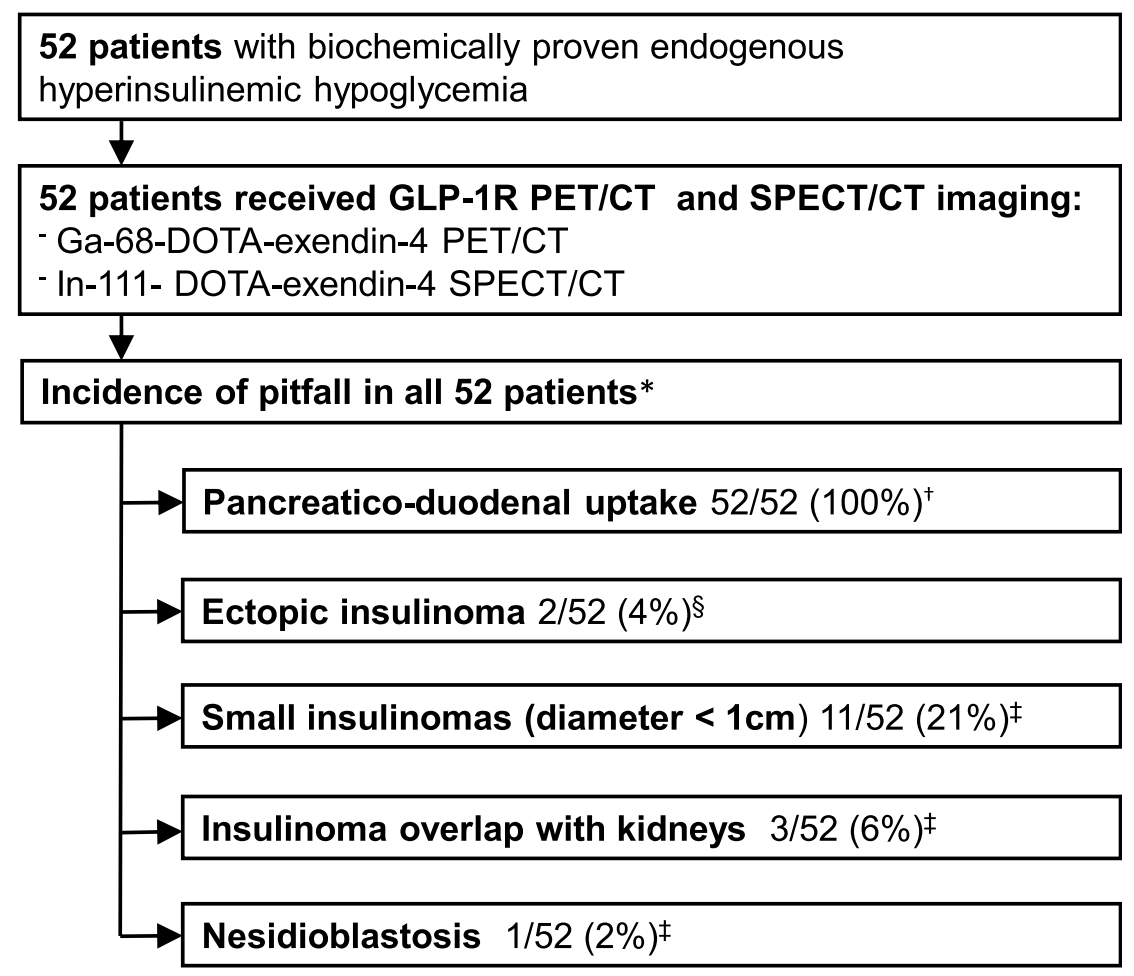

FIGURE 1. Participant selection and outcome flow figure. *The incidence of pitfalls is given as absolute number and percentage in a per-patient analysis. †Based on the fact that all duodenal Brunner glands of the duodenum exhibit uptake in a variable degree. §Reference standard was at least 2 repetitive MRI scans and follow-up of neuroglycopenic symptoms. $\ddagger$ Reference standard was surgery with histopathological/immunohistological evaluation.

\begin{abstract}
${ }^{111}$ In-DOTA-exendin-4, 4 and 72 hours postinjection, in a randomized crossover order within 3 to 4 days (Fig. 1). The reference standard was successful surgery with histopathological/immunohistochemical confirmation of an insulinoma. In 2 patients who refused surgery, 2 repetitive MRI scans with follow-up of neuroglycopenic symptoms were used as reference standard.

Detailed information about synthesis and labeling of ${ }^{68} \mathrm{Ga}-$ DOTA-exendin-4 and ${ }^{111}$ In- DOTA-exendin-4, co-administration of glucose infusion, as well as scanning information, has been published elsewhere. ${ }^{2}$
\end{abstract}

\section{Reading and Image Analysis}

All SPECT/CT and PET/CT images were analyzed using a vendor-provided analysis software package (Syngo.via; Siemens Medical Solutions, Malvern, PA).

For localizing insulinomas, SPECT/CT (4 and 72 hours postinjection) and PET/CT readings were previously carried out in a randomized, blinded manner by 3 board-certified nuclear medicine physicians. All readers were unaware of the patient's identity, other imaging results, and the patient's clinical history and assessed images independent from each other.

On visual analysis, abnormal uptake (focal or segmental) was determined as positive for an insulinoma or nesidioblastosis when the lesion exhibited a nonphysiological increased uptake that was clearly visible and discernible from background activity. False reading was defined as follows: (1) false-negative reading: insulinoma/ nesidioblastosis missed by readers and (2) false-positive reading was defined as physiological uptake interpreted by readers as insulinoma or nesidioblastosis.
Quantitative analysis was performed by a nonblinded nuclear medicine physician:

For the calculation of insulinoma-to-background ratios, duodenum-to-background ratios, and insulinoma-to-duodenum ratios, spherical volumes of interest were placed over the regions of normal pancreatic tissue (background), first and second parts of the duodenum (pancreaticoduodenal uptake), and areas of focal or segmental increased pancreatic uptake (insulinomas, nesidioblastosis) on PET/ $\mathrm{CT}$ and SPECT/CT scans.

The same nonblinded nuclear medicine physician assessed scans and clinical information to determine the size and localization of insulinomas, as well as the presence of nesidioblastosis.

\section{RESULTS}

Figure 1 illustrates the participant selection and outcome flow figure, whereas the main results are summarized in Table 1. The physiological ${ }^{111}$ In-exendin-4 and ${ }^{68}$ Ga-DOTA-exendin-4 distribution of a patient with a normal whole-body scintigraphy, SPECT/CT, and PET/CT scan is shown in Figure 2. Independent reading of GLP-1R scans revealed false-negative results in 7.1\% of PET/CT and $20.5 \%$ of SPECT/CT scans. There was no falsepositive reading. By further evaluation of false-negative reading results, the following pitfalls could be identified: peripancreatic uptake, small lesions, kidney overlap, and diffuse increased pancreatic uptake (Table 1).

\section{Peripancreatic Uptake}

Specific radiotracer accumulation in GLP-1R-positive Brunner glands located in the proximal duodenum (pancreaticoduodenal uptake) was the most common pitfall (100\% incidence) and was 
TABLE 1. Incidence of the Respective Pitfall in 52 Patients With EHH

\begin{tabular}{|c|c|c|c|c|c|}
\hline Pitfall & $\begin{array}{l}\text { Incidence } \\
\text { of Pitfall* }\end{array}$ & $\begin{array}{c}\text { PET/CT: } \\
\text { False Readings/ } \\
\text { Total No. Readings } \dagger\end{array}$ & Type of False Reading & $\begin{array}{c}\text { SPECT/CT: } \\
\text { False Readings/ } \\
\text { Total No. of Readings } \dagger\end{array}$ & $\begin{array}{c}\text { Type of False } \\
\text { Reading }\end{array}$ \\
\hline \multicolumn{6}{|l|}{ Peripancreatic uptake } \\
\hline Pancreaticoduodenal uptake & $52 / 52 \dagger(100 \%)$ & $1 / 156(0.6 \%)$ & $\mathrm{FN}$ & $14 / 156(9.0 \%)$ & $\mathrm{FN}$ \\
\hline Ectopic insulinoma & $2 / 52(4 \%)$ & $0 / 156(0 \%)$ & None & $4 / 156(2.6 \%)$ & $\mathrm{FN}$ \\
\hline \multicolumn{6}{|l|}{ Small lesions (diameter $<1 \mathrm{~cm}$ ) } \\
\hline Small insulinomas & $11 / 52(21 \%)$ & $3 / 156(1.9 \%)$ & $\mathrm{FN}$ & $8 / 156(5.1 \%)$ & $\mathrm{FN}$ \\
\hline \multicolumn{6}{|l|}{ Kidney overlap } \\
\hline Insulinoma overlap with kidneys & $3 / 52(6 \%)$ & $3 / 156(1.9 \%)$ & $\mathrm{FN}$ & $7 / 156(4.5 \%)$ & $\mathrm{FN}$ \\
\hline \multicolumn{6}{|l|}{ Diffuse increased pancreas uptake } \\
\hline Nesidioblastosis & $1 / 52(2 \%)$ & $1 / 156(0.6 \%)$ & $\mathrm{FN}$ & $3 / 156(1.9 \%)$ & $\mathrm{FN}$ \\
\hline Total & $52 / 52(100 \%)$ & $8 / 156(5.1 \%)$ & $\mathrm{FN}$ & $32 / 156(20.5 \%)$ & $\mathrm{FN}$ \\
\hline
\end{tabular}

A

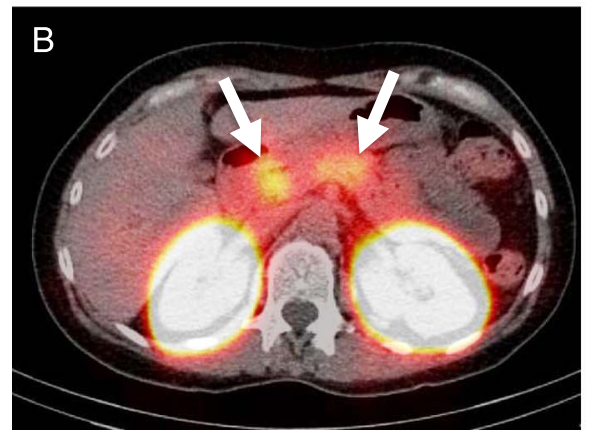

C

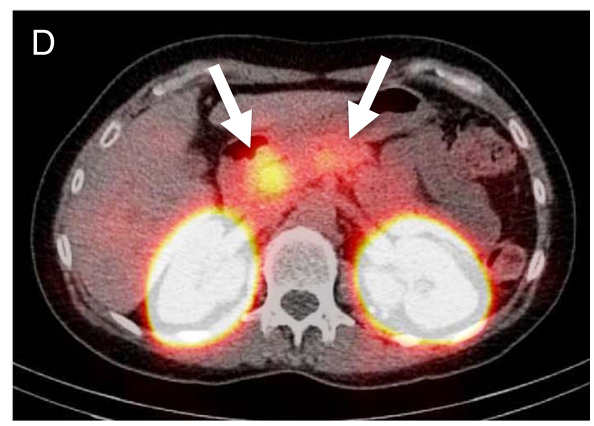

FIGURE 2. Normal scan (patient 11): full body scan acquired 72 hours after the injection of ${ }^{111}$ In-DOTA-exendin-4 shows the physiological distribution with moderate uptake in the salivary glands, diffuse uptake in the pancreas, and renal radiotracer excretion through the kidneys with subsequent accumulation in the bladder (A). Transaxial SPECT/CT acquired 72 hours after the injection of ${ }^{111}$ In-DOTA-exendin-4 shows physiological, diffuse uptake in the pancreas (arrows) and renal radiotracer accumulation (B). Maximum intensity projection image 2.5 hours after injection of ${ }^{68}$ Ga-DOTA-exendin-4 shows physiological radiotracer distribution with moderate uptake in the salivary glands, discrete mediastinal blood pool activity, diffuse uptake in the pancreas, and renal excretion through the kidneys with subsequent accumulation in the bladder (C). Representative transaxial ${ }^{68} \mathrm{Ga}$-DOTA-exendin-4 PET/CT image shows the physiological moderate radiotracer uptake in the pancreas (arrows) and renal accumulation (D). 

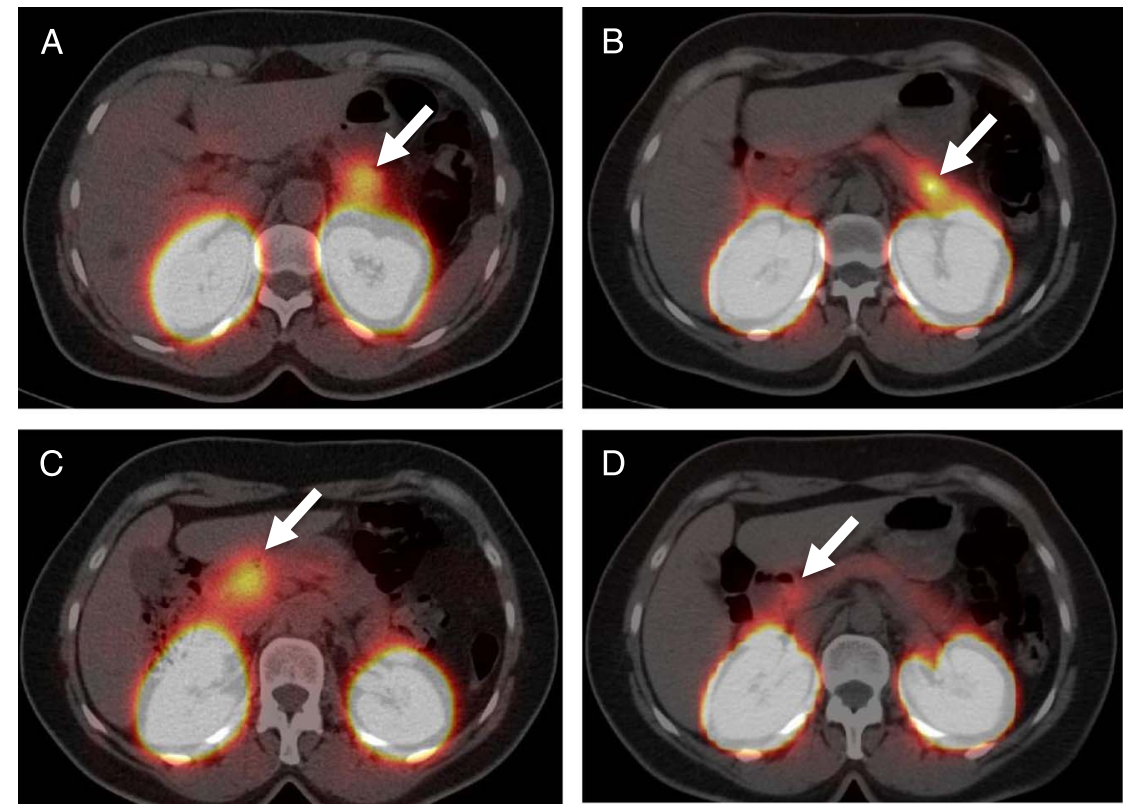

FIGURE 3. Insulinoma and pancreaticoduodenal uptake (patient 2): transaxial SPECT/CT depicts a histopathologically proven insulinoma in the pancreatic tail (arrow) with maximum count of 20.5 (A). Transaxial PET/CT shows a histopathologically proven insulinoma in the pancreatic tail (arrow) with an SUVmax of 10.4 (B). Transaxial SPECT/CT of the same patient depicts a physiological pancreaticoduodenal uptake (arrow) in Brunner glands with maximum count of 21.5 (C). Transaxial PET/CT shows a physiological pancreaticoduodenal uptake (arrow) in Brunner glands with an SUVmax of 5.3 (D).

therefore almost always the reason for peripancreatic uptake of radiolabeled exendin-4 (Fig. 3). Two ectopic insulinomas were identified as the only exception (Fig. 4). Pancreaticoduodenal uptake did mislead the readers in their interpretation especially in ${ }^{111}$ In-DOTA-exendin-4 SPECT/CT scans resulting in falsenegative reading results (falsely interpreted as physiological uptake in Brunner glands instead of insulinomas): in $0.6 \%(1 / 156)$ false-negative readings with PET/CT and 9.0\% (14/156) falsenegative readings with SPECT/CT (Table 1). Because reading was done by 3 nuclear medicine physicians, a total of 156 readings were done ( 3 readers $\times 52$ patients $=156$ readings $)$.

$\mathrm{PET} / \mathrm{CT}$ performed much better in this situation, correlating with the quantitative analysis, which revealed a median insulinomato-duodenum ratio of 2.95 for PET/CT and only 1.72 and 1.84 for SPECT/CT at 4 and 72 hours (Table 2 and Fig. 3).

Table 2 shows the comparison of uptake ratios between 2.5 hours PET/CT, 4 hours SPECT/CT, and 72 hours SPECT/CT.

\section{Small Lesions}

Eleven patients had a small insulinoma (diameter $<1 \mathrm{~cm}$ ). Three $(1.9 \%)$ of 156 false-negative readings occurred in PET/CT in comparison to SPECT/CT with $8(5.1 \%)$ of 156 false-negative readings (Table 1 and Fig. 4). Three of those 11 patients have genetically proven MEN-1 (Fig. 5).

\section{Kidney Overlap}

Three insulinomas were located in the distal pancreatic tail, close to the kidney. Three (1.9\%) of 156 false-negative readings occurred with PET/CT in comparison to SPECT/CT with 7 (4.5\%) of 156 false-negative readings (Fig. 6).

\section{Diffuse Increased Pancreatic Uptake}

One patient had a histopathologically proven nesidioblastosis. Only 1 PET/CT reader (1/156 [0.6\%]) read false-negative, whereas
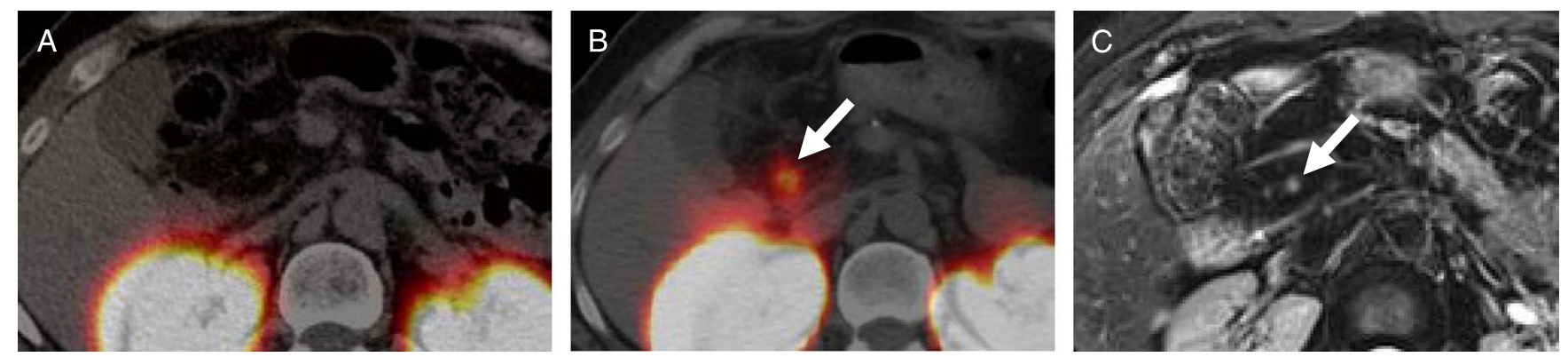

FIGURE 4. A small, ectopic insulinoma (patient 12): transaxial SPECT/CT acquired 72 hours after the injection of ${ }^{111}$ In-DOTAexendin-4 shows physiological renal tracer accumulation but no pathological uptake in the abdomen (A). Transaxial PET/CT 2.5 hours after injection of ${ }^{68} \mathrm{Ga}$-DOTA-exendin-4 of the same patient shows a clear focal uptake in the peripancreatic fat consistent with an insulinoma (arrow) (B). Transaxial T2 weighted MRI shows a 5-mm measuring lesion (arrow) in the peripancreatic fat in correlation with previously performed PET/CT (C). 
TABLE 2. Comparison of Uptake Ratios Between 2.5 Hours PET/CT, 4 Hours SPECT/CT, and 72 Hours SPECT/CT

\begin{tabular}{lccr}
\hline Imaging Modality & Insulinoma-to-Background Ratio & Duodenum-to-Background Ratio & Insulinoma-to-Duodenum Ratio \\
\hline $2.5 \mathrm{~h} \mathrm{PET/CT}$ & $3.45(2.26-5.58)$ & $1.17(0.98-1.59)$ & $2.95(1.68-4.76)$ \\
$4 \mathrm{~h} \mathrm{SPECT/CT}$ & $2.17(1.62-3.95)$ & $1.26(0.78-2.51)$ & $1.72(1.01-2.58)$ \\
$72 \mathrm{~h} \mathrm{SPECT/CT}$ & $2.19(1.53-3.20)$ & $1.19(0.79-1.60)$ & $1.84(1.26-3.36)$ \\
\hline
\end{tabular}

Data are expressed in median (IQR).

all SPECT/CT readers (3/156 [1.9\%]) were not able to identify the nesidioblastosis (Fig. 7).

\section{DISCUSSION}

The increasing availability of GLP-1R imaging with PET tracers such as ${ }^{68}$ Ga-DOTA-exendin-4 and ${ }^{68}$ Ga-NODAGAexendin-4 or SPECT tracers such as ${ }^{99 \mathrm{~m}}$ Tc-HYNIC-exendin-4, ${ }^{111}$ In-DOTA-exendin-4, or ${ }^{111}$ In-DTPA-exendin-4 raises the need for awareness of potential pitfalls in image interpretation.

In this post hoc analysis of our prospective study involving 52 patients, we were able to identify several potential pitfalls, which can be classified into 2 major groups: scan with peripancreatic uptake and scan with missing focal pancreas uptake.

\section{Scan With Peripancreatic Uptake}

Accumulation of GLP-1R-specific radiotracers in Brunner glands is responsible for the physiological peripancreatic uptake. ${ }^{9}$ It is the most common pitfall as Brunner glands are always present and accountable for the pancreaticoduodenal uptake, which can cause confusion for the readers: Brunner glands versus insulinoma.

Brunner glands including Brunner glands hyperplasia have been shown in ex vivo immunohistochemistry staining and autoradiography to express GLP-1R in high density, making them relevant targets for GLP-1R specific radiotracers. ${ }^{8,9}$ These glands are located in the proximal duodenum and secrete a protective layer against gastric acid and digestive enzymes. ${ }^{10} \mathrm{Christ}$ et $\mathrm{al}^{11}$ first reported in 2013 that Brunner glands may be an explanation for false-positive results in GLP-1R SPECT/CT. False-positive results due to pancreaticoduodenal uptake possibly lead to relevant clinical consequences, as lesions located in the pancreatic head are mostly resected through duodenopancreatectomy (Whipple surgery), in case enucleation is not possible. Therefore, a high diagnostic confidence in the evaluation of focal pancreaticoduodenal uptake is desirable. In insulinomas located in the pancreatic head, GLP-1R $\mathrm{PET} / \mathrm{CT}$ encountered far fewer false readings in comparison to SPECT/CT ( $0.6 \%$ vs $9.0 \%$ false readings, respectively). In all these

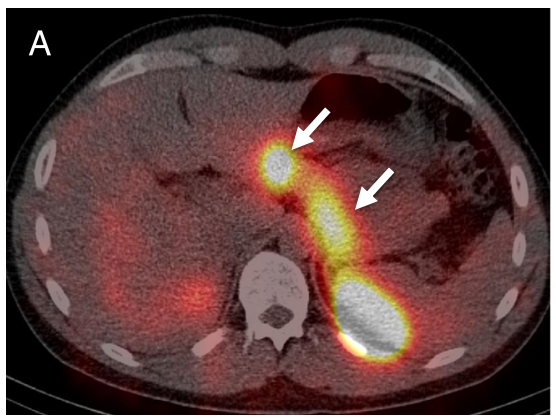

false readings, insulinomas located in the pancreatic head were misinterpreted as unspecific Brunner glands uptake and were therefore missed (false-negative reading).

The lower rate of false readings in PET/CT can be explained by the results of quantitative measures. Although insulinoma-tobackground ratio was higher than duodenum-to-background ratio in both PET/CT and SPECT/CT, the difference in ratios was clearly less pronounced in SPECT/CT, which had an influence in the reading confidence in pancreaticoduodenal uptakes.

Consequently, if SPECT/CT is used in GLP-1R imaging, morphological correlation, preferably with $\mathrm{MRI},{ }^{12}$ is mandatory to reduce misinterpretation, especially if lesions are suspected in the pancreatic head.

Ectopic insulinomas, although extremely rare, ${ }^{13}$ can be another cause for peripancreatic uptake as they can occur anywhere in the peripancreatic fat or in the duodenal wall ${ }^{14}$ (Fig. 4).

\section{Scan With Missing Focal Pancreatic Uptake}

Biochemical evidence for an insulinoma but absence of focal radiotracer uptake in the pancreas might occur in the following situations: (1) insulinoma is not localized in the pancreas (ectopic insulinoma); (2) insulinomas are too small to be detected with PET/CT or SPECT/CT; (3) insulinomas overlap with kidneys; and (4) $\mathrm{EHH}$ is caused by beta-cell hyperplasia (nesidioblastosis) and not by an insulinoma.

(1) Ectopic insulinomas: Although extremely rare, ${ }^{13}$ ectopic insulinomas can be the reason for missing focal uptake in the pancreas as they can occur anywhere in the peripancreatic fat or in the duodenal wall. ${ }^{14}$ In our study, 2 patients with high suspicion for an ectopic insulinoma were identified with ${ }^{68} \mathrm{Ga}-\mathrm{DOTA}$ exendin-4 PET/CT. One of those 2 patients received extensive diagnostic workup since 2010 , leading to a (blind) pancreatic left resection, which did not reveal any suspicious lesion. Also, GLP-1R SPECT/CT performed 2 years later did not reveal any suspicious lesion. However, both ${ }^{68} \mathrm{Ga}$-DOTA-exendin-4 PET/

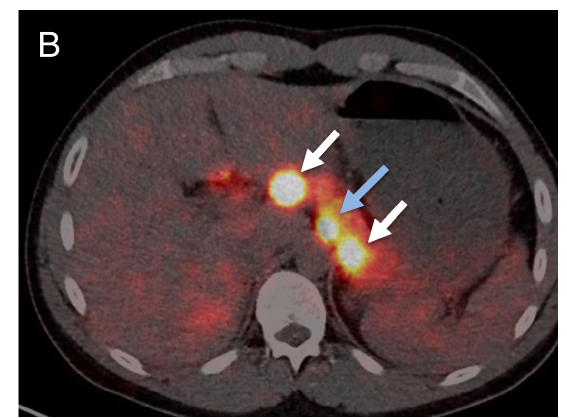

FIGURE 5. Multiple insulinomas including 1 small insulinoma (patient 46): transaxial SPECT/CT of a patient with MEN-1 and $\mathrm{EHH}$ acquired 72 hours after the injection of ${ }^{111}$ In-DOTA-exendin-4 depicts 2 lesions in the pancreas (small arrows) (A). Histology revealed 2 insulinomas with a diameter of 17 and $11 \mathrm{~mm}$. Transaxial PET/CT 2.5 hours after injection of ${ }^{68} \mathrm{Ga}$-DOTAexendin- 4 of the same patient shows 1 additional lesion (large arrow) in the pancreatic tail consistent with an additional, histopathologically proven insulinoma with a diameter of $6 \mathrm{~mm}$ (B). 

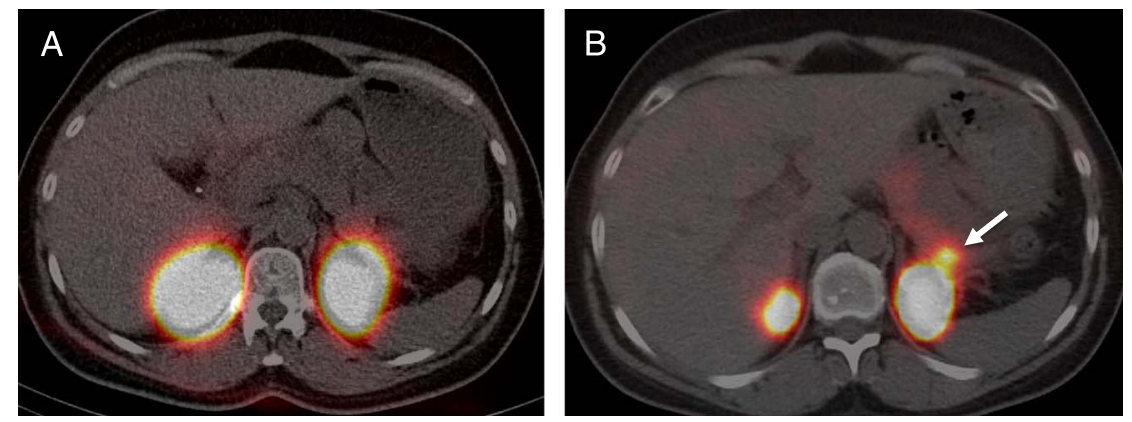

FIGURE 6. Insulinoma overlap with kidneys (patient 6): ${ }^{111}$ In-DOTA-exendin-4 SPECT/CT showed radiotracer accumulation only in the kidneys but no evidence of an insulinoma (A). ${ }^{68} \mathrm{Ga-DOTA-exendin-4} \mathrm{PET/CT}$ of the same patient showed strong focal uptake within the distal pancreatic tail (arrow) consistent with a histologically proven insulinoma (B).

CTs performed in January 2014 and August 2016 showed an intensive focal uptake in a small lesion in the peripancreatic fat anterior to the pancreatic body compatible with an insulinoma. The other patient was diagnosed with EHH in 2004, henceforth received extensive diagnostic workup, including ASVS in 2009, distal pancreatectomy in 2012, and repeat explorative laparotomy in 2014. Glucagon-like peptide-1 receptor PET/CT in 2014 revealed a clear focal uptake in the peripancreatic fat just below the pancreatic head (Fig. 4). Unfortunately, both patients refused additional surgery due to previous unsuccessful surgery. The standard for comparison in both patients was 2 positive repetitive MRI scans and continuation of neuroglycopenic symptoms. Ectopic insulinomas should be considered as a differential diagnosis in patients with EEH and missing evidence of focal radiotracer uptake in the pancreas. In such cases, the peripancreatic region should be screened for areas of focal increased uptake, which is a sign for the presence of an ectopic insulinoma.

(2) Small insulinomas: Despite not being a real pitfall, the most common cause for missing ${ }^{68} \mathrm{Ga}$-exendin-4 or ${ }^{111}$ In-exendin-4 uptake in the pancreas is the small size of the insulinoma. Especially lesions with a diameter of less than $10 \mathrm{~mm}$ are a major problem as they are often below the scanner sensitivity of GLP$1 \mathrm{R}$ imaging. Eleven $(21.2 \%)$ of 52 patients had an insulinoma with a diameter of $10 \mathrm{~mm}$ or less; $1.9 \%$ false-negative readings occurred with PET/CT in comparison to $5.1 \%$ false-negative readings for SPECT/CT. The better performance of PET/CT is attributed to the higher spatial resolution and sensitivity of PET/CT, which is able to detect insulinomas up to a size of $4 \mathrm{~mm} .^{5}$ This is also true in patients with MEN-1 who often have multiple small insulinomas. ${ }^{5}$ In the case of negative GLP-1R scan and negative morphological imaging, a repeat GLP-1R scan after 1 or 2 years should be considered as possible lesion growth may increase the probability that the occult tumor will be depicted. ${ }^{15}$

(3) Insulinoma overlap with kidneys: Localization of insulinomas close to the kidney can be problematic, as the excretion of GLP$1 \mathrm{R}$ specific radiotracers is mainly via the urinary tract. ${ }^{16}$ Especially lesions located close to the kidneys can be masked due to the high renal uptake. ${ }^{17}$ The inherent high partial volume effect is thus a known limitation and potential pitfall of GLP-1R imaging. The aforementioned effect is less pronounced in PET/CT than in SPECT/CT owing to the higher spatial resolution of $\mathrm{PET} / \mathrm{CT},{ }^{18}$ which reflects in the lower false-negative reading rate of PET/CT (1.9\%) in comparison to SPECT/CT (4.5\%). Careful inspection in the kidney contours can increase detectability of insulinomas localized close to the kidney, as a characteristic bulge within the contour might be identified (Fig. 6). In case of negative GLP-1R imaging, correlation with MRI or CT is recommended in order to identify a possible insulinoma close to the kidneys. If such a correlation is not successful, and the patient has persistent hypoglycemia, a considerable reduction in renal GLP-1R tracer uptake can be accomplished through the prior infusion of Gelofusion, a plasma expander known to induce lowmolecular-weight proteinuria. ${ }^{17,19,20}$ The off-label use of this infusion may improve the detection of lesions close to the kidneys as previously published. ${ }^{17,21}$

In case of all noninvasive imaging and localization procedures being negative, selective intra-ASVS should still be considered.

(4) Nesidioblastosis: Beta-cell hyperplasia (nesidioblastosis) is a differential diagnosis of EHH. ${ }^{22,23}$ In this situation, the clinical distinction to EHH caused by insulinomas is normally
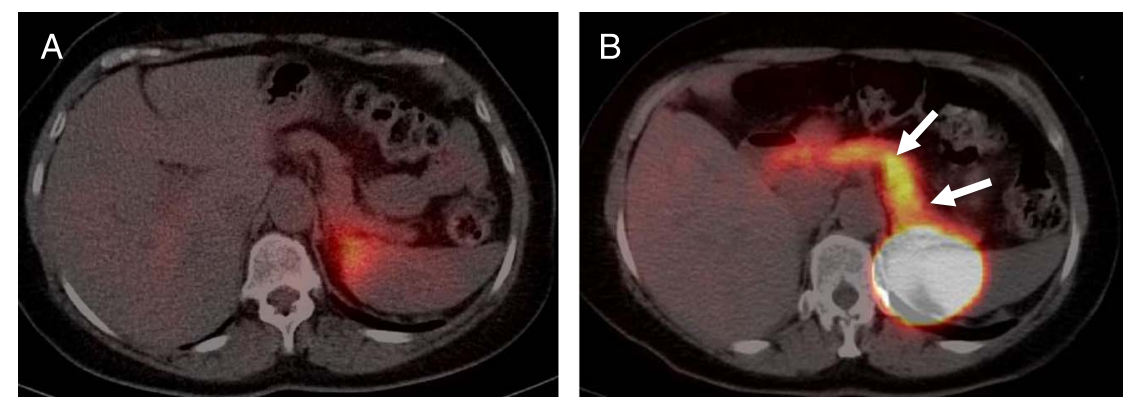

FIGURE 7. Nesidioblastosis (patient 5): transaxial SPECT/CT acquired 72 hours after the injection of ${ }^{111}$ In-DOTA-exendin-4 shows no pathological radiotracer accumulation (A). Transaxial PET/CT of the same patient acquired 2.5 hours after injection of ${ }^{68} \mathrm{Ga}$-DOTA-exendin-4 shows a pathological radiotracer uptake in the pancreatic tail consistent with a histopathologically proven nesidioblastosis (B). 
not possible. In addition, morphological imaging using CT, MRI, or endoscopic ultrasound is not helpful in the detection of nesidioblastosis. Often only ASVS is helpful to identify/ localize focal nesidioblastosis, but comes along with the associated risk of an invasive procedure. In a recent case report, we were able to show that GLP-1R PET/CT may be able to localize focal or segmental areas of nesidioblastosis. ${ }^{6}$ In this patient, GLP-1R PET/CT indicated the area of diffuse uptake in the pancreatic tail. Based on these findings, a left-sided pancreatectomy was performed, and histology confirmed the presence of a nesidioblastosis. All 3 SPECT readers were false-negative in this patient. Therefore, nesidioblastosis should always be considered as a differential diagnosis in patients with $\mathrm{EHH}$ and missing evidence of focal radiotracer uptake. In such a case, the pancreas should be screened for areas of diffuse increased ${ }^{68}$ Ga-exendin-4 uptake, which may be an indication for the presence of beta-cell hyperplasia (nesidioblastosis).

This study has limitations. (1) Evaluation of pitfalls was performed only with DOTA-exendin-4 tracers. Other clinically evaluated exendin-4 derivatives, such as [Lys ${ }^{40}$ (Ahx-HYNIC- ${ }^{99 m}$ Tc/EDDA) $\mathrm{NH}_{2}$ ]-exendin- $4,{ }^{24}{ }^{68} \mathrm{Ga}-\mathrm{NODAGA}$-exendin-4, ${ }^{25}$ and ${ }^{111}$ In-DTPAexendin- $4,{ }^{1}$ as well as preclinically evaluated tracers, ${ }^{26}$ have not been evaluated in this study. Pitfalls may vary in these derivatives. (2) Patients with signs of malignancy on conventional imaging were excluded in this study, although few case reports exist that showed GLP-1R imaging is feasible and detects malignant insulinomas. ${ }^{3}$ As opposed to benign insulinomas, malignant insulinomas often lack GLP-1 receptors and conversely frequently express the somatostatin receptor type $2 .^{4}$

\section{CONCLUSIONS}

Peripancreatic uptake, small size of an insulinoma, insulinoma overlap with kidneys, and the presence of nesidioblastosis are potential pitfalls in GLP-1R imaging, which can lead to false reading results.

\section{ACKNOWLEDGMENTS}

The authors thank all the patients who participated in the trial, the referring physicians and the local investigators who contributed to the trial, and the technicians who did the labeling and the scans. They especially thank Prof Aurel Perren, Institute of Pathology, University Bern, Switzerland, for pathological review and Astrid Roesler, Clinical Trial Unit, Department of Clinical Research, University Hospital Basel, and University of Basel, Switzerland, for monitoring the study.

\section{REFERENCES}

1. Christ E, Wild D, Ederer S, et al. Glucagon-like peptide-1 receptor imaging for the localisation of insulinomas: a prospective multicentre imaging study. Lancet Diabetes Endocrinol. 2013;1:115-122.

2. Antwi K, Fani M, Heye T, et al. Comparison of glucagon-like peptide-1 receptor (GLP-1R) PET/CT, SPECT/CT and 3T MRI for the localisation of occult insulinomas: evaluation of diagnostic accuracy in a prospective crossover imaging study. Eur J Nucl Med Mol Imaging. 2018;45:2318-2327.

3. Eriksson O, Velikyan I, Selvaraju RK, et al. Detection of metastatic insulinoma by positron emission tomography with [(68)Ga]exendin-4 - a case report. J Clin Endocrinol Metab. 2014;99:1519-1524.

4. Wild D, Christ E, Caplin ME, et al. Glucagon-like peptide-1 versus somatostatin receptor targeting reveals 2 distinct forms of malignant insulinomas. J Nucl Med. 2011;52:1073-1078.
5. Antwi K, Nicolas G, Fani M, et al. ${ }^{68}$ Ga-exendin-4 PET/CT detects insulinomas in patients with endogenous hyperinsulinemic hypoglycemia in MEN-1. J Clin Endocrinol Metab. 2019;104:5843-5852.

6. Christ E, Wild D, Antwi K, et al. Preoperative localization of adult nesidioblastosis using ${ }^{68} \mathrm{Ga}$-DOTA-exendin-4-PET/CT. Endocrine. 2015; 50:821-823.

7. Wild D, Mäcke H, Christ E, et al. Glucagon-like peptide 1-receptor scans to localize occult insulinomas. $N$ Engl J Med. 2008;359:766-768.

8. Körner M, Stöckli M, Waser B, et al. GLP-1 receptor expression in human tumors and human normal tissues: potential for in vivo targeting. $J \mathrm{Nucl}$ Med. 2007;48:736-743.

9. Hepprich M, Antwi K, Waser B, et al. Brunner's gland hyperplasia in a patient after roux-Y gastric bypass: an important pitfall in GLP-1 receptor imaging. Case Rep Endocrinol. 2020;2020:1-4.

10. Krause WJ. Brunner's glands: a structural, histochemical and pathological profile. Prog Histochem Cytochem. 2000;35:259-367.

11. Christ E, Wild D, Forrer F, et al. Glucagon-like peptide-1 receptor imaging for localization of insulinomas. J Clin Endocrinol Metab. 2009;94: 4398-4405.

12. Zhu L, Xue H, Sun Z, et al. Prospective comparison of biphasic contrastenhanced CT, volume perfusion CT, and 3 tesla MRI with diffusionweighted imaging for insulinoma detection. J Magn Reson Imaging. 2017; 46:1648-1655.

13. La Rosa S, Pariani D, Calandra C, et al. Ectopic duodenal insulinoma: a very rare and challenging tumor type. Description of a case and review of the literature. Endocr Pathol. 2013;24:213-219.

14. Komminoth P, Heitz PU, Roth J. Human insulinomas: clinical, cellular, and molecular aspects. Endocr Pathol. 1999;10:269-281.

15. Cuthbertson DJ, Banks M, Khoo B, et al. Application of Ga(68) -DOTAexendin-4 PET/CT to localize an occult insulinoma. Clin Endocrinol (Oxf). 2016;84:789-791.

16. Wild D, Béhé M, Wicki A, et al. [Lys40(Ahx-DTPA- ${ }^{111}$ In) $\mathrm{NH}_{2}$ ] exendin-4, a very promising ligand for glucagon-like peptide-1 (GLP-1) receptor targeting. J Nucl Med. 2006;47:2025-2033.

17. Antwi K, Nicolas G, Fani M, et al. Volume replacement fluid demarks benign Insulinoma with ${ }^{68} \mathrm{Ga}$-DOTA-exendin-4 PET/CT. Clin Nucl Med. 2019;44:e347-e348.

18. Martin WH, Delbeke D, Patton JA, et al. Detection of malignancies with SPECT versus PET, with 2-[fluorine-18]fluoro-2-deoxy-D-glucose. Radiology. 1996;198:225-231.

19. Vegt E, Wetzels JF, Russel FG, et al. Renal uptake of radiolabeled octreotide in human subjects is efficiently inhibited by succinylated gelatin. $J \mathrm{Nucl}$ Med. 2006;47:432-436.

20. van Eerd JE, Vegt E, Wetzels JF, et al. Gelatin-based plasma expander effectively reduces renal uptake of ${ }^{111}$ In-octreotide in mice and rats. J Nucl Med. 2006; $47: 528-533$

21. Buitinga M, Jansen TJP, van der Kroon I, et al. Succinylated gelatin improves the theranostic potential of radiolabeled exendin-4 in insulinoma patients J Nucl Med. 2018;60:812-816.

22. Jabri AL, Bayard C. Nesidioblastosis associated with hyperinsulinemic hypoglycemia in adults: review of the literature. Eur J Intern Med. 2004;15: $407-410$.

23. Anlauf M, Wieben D, Perren A, et al. Persistent hyperinsulinemic hypoglycemia in 15 adults with diffuse nesidioblastosis: diagnostic criteria, incidence, and characterization of beta-cell changes. Am J Surg Pathol. 2005; 29:524-533.

24. Sowa-Staszczak A, Pach D, Mikołajczak R, et al. Glucagon-like peptide-1 receptor imaging with [Lys40(Ahx-HYNIC-99m Tc/EDDA) $\mathrm{NH}_{2}$ ]-exendin-4 for the detection of insulinoma. Eur J Nucl Med Mol Imaging. 2013;40 524-531.

25. Boss $M$, Buitinga $M$, Jansen TJ, et al. PET-based dosimetry of ${ }^{68} \mathrm{Ga}-$ NODAGA-exendin-4, a tracer for $\beta$-cell imaging. J Nucl Med. 2020;61: 112-116.

26. Wild D, Wicki A, Mansi R, et al. Exendin-4-based radiopharmaceuticals for glucagonlike peptide-1 receptor PET/CT and SPECT/CT. J Nucl Med. 2010 51:1059-1067. 